\title{
The Effect of Acute Stress on Esophageal Motility and Gastroesophageal Reflux in Healthy Humans
}

\author{
Hong Sub Lee, ${ }^{1,2}$ Chung Kyun Noh, ${ }^{1}$ and Kwang Jae Lee ${ }^{1 *}$ \\ ${ }^{1}$ Department of Gastroenterology, Ajou University School of Medicine, Suwon, Gyeonggi-do, Korea; and ${ }^{2}$ Department of Gastroenterology, \\ Seonam University College of Medicine, Myongji Hospital, Goyang; Gyeonggi-do, Korea
}

\section{Background/Aims}

Little research has been done to evaluate the effect of stress in exacerbating the symptoms associated with gastroesophageal reflux (GER). We aimed to investigate the effect of acute stress on esophageal motility and GER parameters in healthy volunteers.

\section{Methods}

A total of 10 (M:F = 8:2, median age 34 years-old) healthy volunteers without any recurrent gastrointestinal symptoms participated in this study. They underwent esophageal high-resolution manometry with 10 wet swallows (Experiment I) and esophageal impedance$\mathrm{pH}$ monitoring (Experiment II) in the basal period and in the stress period. In the stress period, either real stress or sham stress was given in a randomized cross-over design. The stress scores, symptom severity, and pulse rates were measured.

\section{Results}

The stress scores and the severity of nausea were significantly greater under real stress, compared with sham stress. The percentages of weak, failed, rapid, premature, and hyper-contractile contractions were not significantly altered during real stress and during sham stress, compared with the basal period. The median resting pressure of the lower esophageal sphincter and distal contractile integral of esophageal contractions did not differ in the stress period, compared with the basal period. Contractile front velocity and distal latency of esophageal peristaltic contractions were significantly changed during real stress, which was not observed during sham stress. GER parameters were not significantly altered during real stress and during sham stress.

\section{Conclusion}

Although acute auditory and visual stress seems to affect esophageal body motility, it does not induce significant motor abnormalities or increase GER in healthy humans.

(J Neurogastroenterol Motil 2017;23:72-79)

\section{Key Words}

Esophageal motility; Gastroesophageal reflux; Stress

Received: July 23, 2016 Revised: October 3, 2016 Accepted: October 18, 2016

(a) This is an Open Access article distributed under the terms of the Creative Commons Attribution Non-Commercial License (http://creativecommons. org/licenses/by-nc/4.0) which permits unrestricted non-commercial use, distribution, and reproduction in any medium, provided the original work is properly cited.

${ }^{*}$ Correspondence: Kwang Jae Lee, MD Department of Gastroenterology, Ajou University Hospital, Ajou University School of Medicine, 164 Worldcup-ro, Yeongtong-gu, Suwon, Gyeonggi-do 16499, Korea

Tel: +82-31-2195102, Fax: +82-31-2195999, E-mail: kjleemd@hotmail.com 


\section{Introduction}

Psychological stress is known to play a crucial role in the pathogenesis of gut motility abnormalities. ${ }^{1,2}$ Previous animal studies have shown that acute psychological or physical stress is associated with motor and sensory abnormalities that can be seen in patients with functional gastrointestinal disorders. ${ }^{3-5}$ Accordingly, emotional stress is believed to be a precipitating factor of gastrointestinal symptoms in patients with functional gastrointestinal disorders. Similarly, evidence suggests that stress plays an important role in the presentation of gastroesophageal reflux disease (GERD). ${ }^{6,7}$ Stress is presumed to exacerbate symptoms associated with gastroesophageal reflux. However, little research has been done to evaluate the effect of stress in exacerbating the symptoms associated with gastroesophageal reflux. Previous studies showed that stress increased symptom perception by enhancing esophageal sensitivity to gastroesophageal reflux. ${ }^{8-10}$ However, it is still debatable whether or not stress influences esophageal motility and gastroesophageal reflux. A few studies regarding the effect of stress on esophageal motility and gastroesophageal reflux have shown controversial results. ${ }^{11-13}$

Diverse models to induce psychological stress have been used in healthy volunteer studies. To induce acute stress, validated dichotomous listening has been commonly used. Such methodology has been validated in inducing psychological and mental stress. ${ }^{14-17}$

In the present study, we aimed to investigate the effect of acute stress on esophageal motility and gastroesophageal reflux parameters in healthy volunteers.

\section{Materials and Methods}

\section{Subjects}

A total of 10 healthy volunteers (2 women and 8 men; median age 34 years; age range 31-37 years) who had no recurrent gastrointestinal symptoms participated in the study. Exclusion criteria included erosive esophagitis on endoscopy; a history of gastrointestinal, neurological or psychiatric disorders; patients on medication that may affect gastrointestinal motility and sensitivity; patients suffering from a severe medical illness; and pregnancy. This study was approved by the institutional review board of Ajou University Hospital. All participants gave informed consent to participate in the study.

\section{Study Design}

This study was performed in a prospective, randomized, crossover design. Subjects participated in 2 separate experiments including the experiment with high-resolution esophageal manometry (HRM) measurement (Experiment I) and the experiment with esophageal multichannel impedance-pH measurement (Experiment II). They underwent esophageal HRM with 10 wet swallows (Experiment I) and esophageal impedance-pH monitoring (Experiment II) in the basal period and in the stress period. In the stress period, either real stress or sham stress was given in a randomized cross-over design. The eligibility of the participants was determined before the start of the experiments.

Subjects were instructed to fast overnight and abstain from caffeine, alcohol, strenuous exercise, and any medication 24 hours

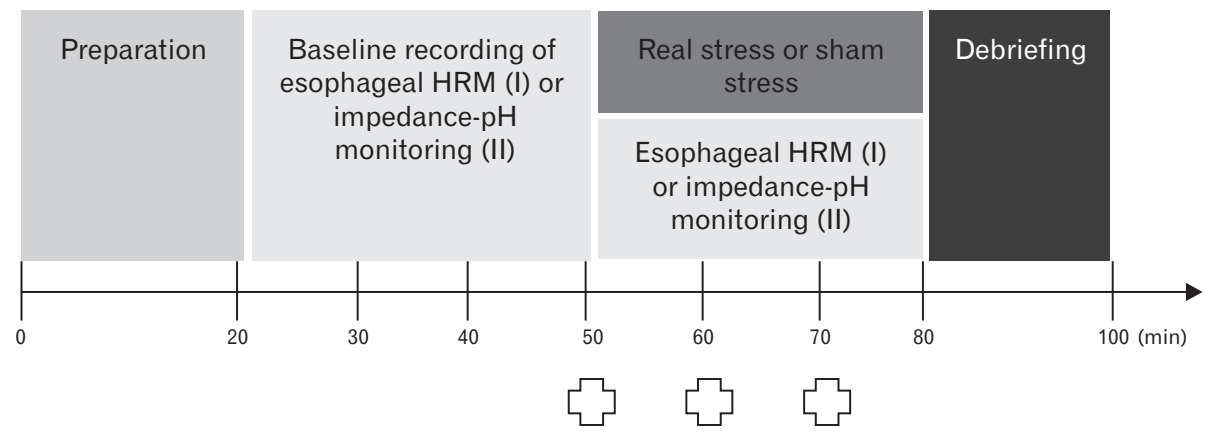

Figure 1. The study protocol. This study was performed in a prospective, randomized, cross-over design. Subjects participated in 2 separate experiments including the experiment with high-resolution esophageal manometry (HRM) measurement (Experiment I) and the experiment with esophageal multichannel impedance-pH measurement (Experiment II). They underwent esophageal HRM with 10 wet swallows (Experiment I) and esophageal impedance-pH monitoring (Experiment II) in the basal period and in the stress period. In the stress period, either real stress or sham stress was given in a randomized cross-over design. ¿3 Assessment of stress levels and symptoms, and measurement of pulse rates. 
before the experiment. All experiments started at the same morning time (9 AM). As depicted in Figure 1, the experiment I included the preparation period (20 minutes), the baseline recording of esophageal HRM (30 minutes), the recording of esophageal HRM under either real stress or sham stress (30 minutes), and the debriefing period (20 minutes). On arrival on each study day, subjects sat in a quiet room and were allowed enough time to relax. Following this, a manometry catheter was inserted transnasally, and the subjects were allowed a period of acclimation to get used to the presence of the catheter. In the baseline recording, they underwent 10 wet swallows. Subsequently, 10 wet swallows were repeated under either real stress or sham stress. The severity of emotional stress and symptoms (heartburn, regurgitation, nausea, epigastric pain, and epigastric burning) perceived during the experiment was assessed using a 10-cm description-anchored visual analogue scale. Subjects were asked to mark this before the onset of stress and after 10 and 20 minutes of stress (Fig. 1). Pulse rates were also measured at the same time.

\section{Real and Sham Stress}

Studies were performed in a quiet, private room. Since the degree of acute auditory stress might be weak to activate the hypothalamic-pituitary-adrenal axis or the autonomic nervous system, visual stress was added to the auditory stress. In the real stress experiment, 2 different types of music were simultaneously delivered using separated stereo earphone channels at a level of $75 \mathrm{~dB}$ while looking at a series of horror pictures. Folk music in a foreign language was played in one ear and 'heavy metal' music in the other ear. In the experiment of sham stress, relaxing music (background piano music) was delivered through both stereo earphone channels while looking at a series of peaceful pictures consisting of baby, pets, nice scenery, and so on.

\section{Esophageal High-resolution Manometry}

HRM was performed using a 22-channel water-perfused silicone esophageal catheter (Solar GI HRM; Medical Measurement Systems, Enschede, The Netherlands). Bi-distilled water circulated through the catheter at a constant flow of $0.6 \mathrm{~mL} / \mathrm{min}$. The perfusion capillary was oriented radially, spaced at $1 \mathrm{~cm}$ in the areas recording the esophagogastric junction and $2 \mathrm{~cm}$ in the areas of the esophageal body. The manometric study was performed with a total of 10 saline $(5 \mathrm{~mL})$ swallows, at 30 second-intervals, in each patient in a semi-recumbent position. Pressure changes were transmitted to external transducers that transform the information in electric signals. Data obtained through the computerized system was analyzed with a specific software (Solar GI HRM; Medical Measurement Systems). The mean resting lower esophageal sphincter (LES) pressure, distal contractile integral, contractile front velocity, and distal latency of esophageal contractions were calculated, based on the criteria of the Chicago classification. ${ }^{18}$

\section{Esophageal Impedance-pH Monitoring}

Combined impedance-pH monitoring was performed using an ambulatory multichannel intraluminal impedance-pH system (Zephyr; Sandhill Scientific Inc, Highlands Ranch, CO, USA). The LES was localized by esophageal manometry. The catheter included four impedance measuring sites located in the distal esophagus at 3, 5, 7 and $9 \mathrm{~cm}$, and 2 impedance measuring sites in the proximal esophagus at 15 and $17 \mathrm{~cm}$ above the LES. An antimony pH sensor was located $5 \mathrm{~cm}$ above the LES. Impedance-detected reflux episodes were classified as "acid" if the $\mathrm{pH}$ sensor recorded a decline in $\mathrm{pH}$ below 4, or as "non-acid" if the $\mathrm{pH}$ remained above 4 during the bolus presence time in the esophagus. The following parameters were obtained from impedance-pH monitoring: total percent of bolus exposure time (BET), total percent of distal esophageal acid exposure time (EAET), esophageal bolus clearance time (BCT; in seconds), and esophageal acid clearance time (ACT; in seconds). BET was defined as the sum of the duration of all reflux episodes (regardless of $\mathrm{pH}$ ) divided by the time monitored. BCT was defined as the time, in seconds, from a $50 \%$ drop in impedance until recovery and above ( $5 \mathrm{~cm}$ above LES). EAET was defined as the percentage of time in which the distal esophageal $\mathrm{pH}$ was less than 4. ACT was defined as the time, in seconds, from $\mathrm{pH}$ below 4 until $\mathrm{pH}$ above 4 .

\section{Statistical Methods}

All data are expressed as mean \pm standard deviation. Paired $t$ test was used for comparing parameters between the baseline and stress periods. Comparison of stress scores, symptom severity values, and pulse rates between real and sham stress experiments was also performed by the paired $t$ test. The $P$-value $<0.05$ was considered statistically significant. The Statistical Package for Social Sciences for Windows version 11 (IBM Corp, Armonk, NY, USA) was used for all analyses.

\section{Results}

\section{Stress Scores, Symptom Severity, and Pulse Rates}

The protocol of stress induction was well-tolerated in all sub- 
jects. The average of stress scores measured 2 times at an interval of 10 minutes in the stress period of Experiment I and Experiment II was significantly greater under real stress, compared with sham stress. The average of nausea scores measured 2 times at an interval of 10 minutes in the stress period of Experiment I and Experiment II was significantly higher under real stress, compared with sham stress. The average scores of other symptoms such as heartburn, regurgitation, epigastric pain, and epigastric burning measured during real stress was not significantly different from that measured during sham stress. The average pulse rates measured during real stress were significantly higher than those measured during sham stress (Table 1).
Table 1. Comparison of Stress Scores, Symptom Severity, and Pulse Rates Between Real and Sham Stress

\begin{tabular}{lccc}
\hline \multicolumn{1}{c}{ Parameters } & Stress & Sham & $P$-value $^{\mathrm{a}}$ \\
\hline Stress score & $5.9 \pm 2.6$ & $2.4 \pm 0.9$ & $<0.001$ \\
Heartburn & $0.2 \pm 0.4$ & 0 & 0.168 \\
Regurgitation & 0 & 0 & $\mathrm{NS}$ \\
Nausea & $2.7 \pm 2.3$ & $0.4 \pm 0.5$ & 0.003 \\
Epigastric pain & $0.7 \pm 0.9$ & $0.6 \pm 0.8$ & 0.343 \\
Epigastric burning & $0.7 \pm 0.8$ & $0.5 \pm 0.7$ & 0.168 \\
Pulse rates (/min) & $88.8 \pm 9.8$ & $77.2 \pm 7.3$ & $<0.001$
\end{tabular}

${ }^{a}$ Paired $t$ test between real and sham stress.

Values are presented as mean \pm standard deviation.

\section{A}

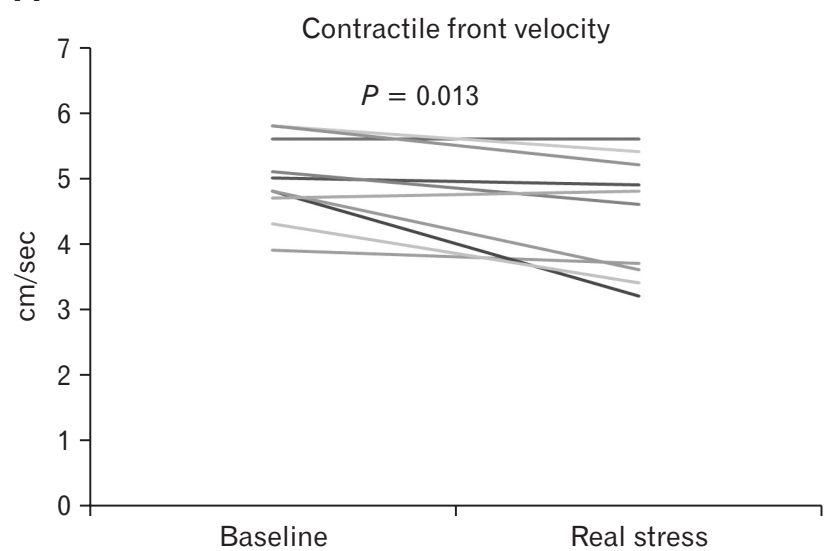

B

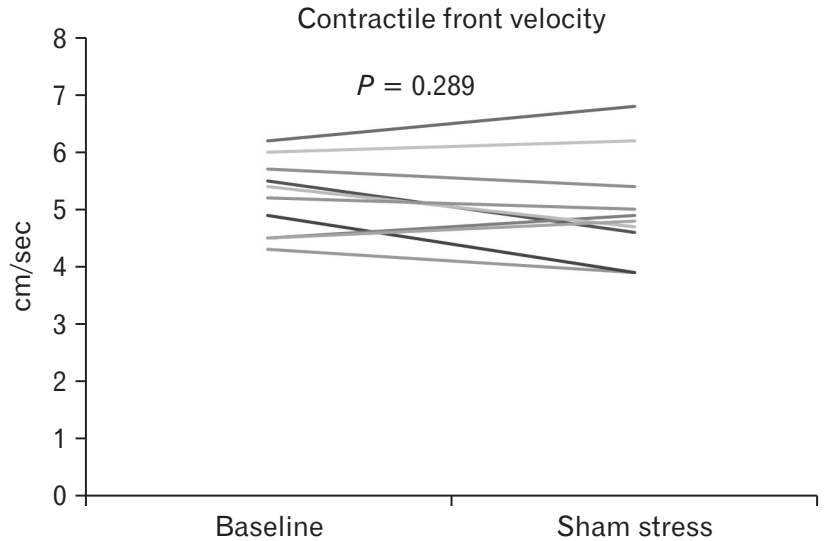

Figure 2. Contractile front velocity of esophageal peristaltic contractions. Contractile front velocity of esophageal peristaltic contractions was significantly decreased during real stress, compared with the baseline period (A), but not during sham stress (B).

\section{A}

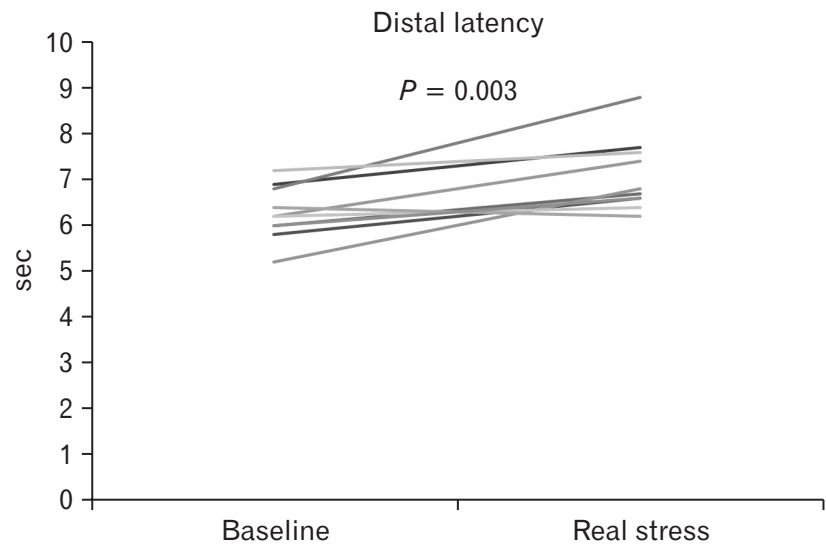

B

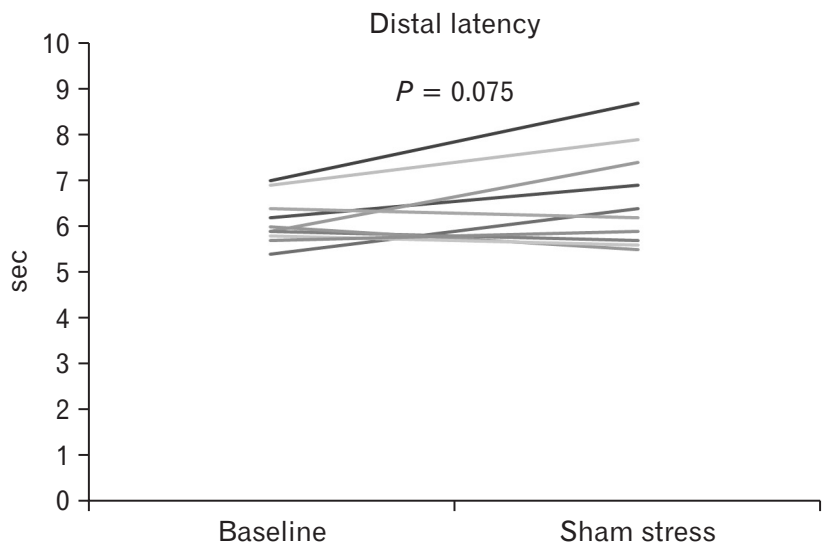

Figure 3. Distal latency of esophageal peristaltic contractions. Distal latency of esophageal peristaltic contractions was significantly increased during real stress, compared with the baseline period (A), but not during sham stress (B). 
Table 2. Parameters of High-resolution Manometry During Real and Sham Stress

\begin{tabular}{|c|c|c|c|}
\hline Parameters & Baseline & Stress period & $P$-value ${ }^{a}$ \\
\hline \multicolumn{4}{|l|}{ Sham stress } \\
\hline Weak contraction $(\%)$ & $7.0 \pm 16.4$ & $8.0 \pm 14.0$ & 0.823 \\
\hline Failed contraction (\%) & 0 & 0 & \\
\hline Rapid contraction $(\%)$ & 0 & $1.0 \pm 3.2$ & 0.343 \\
\hline Premature contraction $(\%)$ & 0 & 0 & \\
\hline Hyper-contractile (\%) & 0 & 0 & \\
\hline Median LES pressure (mmHg) & $20.0 \pm 2.7$ & $20.8 \pm 2.6$ & 0.393 \\
\hline Distal contractile integral $(\mathrm{mmHg} \cdot \mathrm{sec} \cdot \mathrm{cm})$ & $966.4 \pm 484.0$ & $985.7 \pm 519.1$ & 0.840 \\
\hline Contractile front velocity $(\mathrm{cm} / \mathrm{sec})$ & $5.2 \pm 0.6$ & $5.0 \pm 0.9$ & 0.289 \\
\hline Distal latency (sec) & $6.1 \pm 0.5$ & $6.6 \pm 1.1$ & 0.075 \\
\hline \multicolumn{4}{|l|}{ Real stress } \\
\hline Weak contraction $(\%)$ & $3.0 \pm 4.8$ & $14.0 \pm 20.7$ & 0.120 \\
\hline Failed contraction (\%) & 0 & 0 & \\
\hline Rapid contraction (\%) & 0 & $1.0 \pm 3.2$ & 0.343 \\
\hline Premature contraction $(\%)$ & $1.0 \pm 3.2$ & $1.0 \pm 3.2$ & 1.000 \\
\hline Hyper-contractile (\%) & 0 & 0 & \\
\hline Median LES pressure (mmHg) & $22.7 \pm 3.4$ & $21.6 \pm 4.5$ & 0.392 \\
\hline Distal contractile integral $(\mathrm{mmHg} \cdot \mathrm{sec} \cdot \mathrm{cm})$ & $1140.2 \pm 540.7$ & $1200.0 \pm 704.3$ & 0.642 \\
\hline Contractile front velocity $(\mathrm{cm} / \mathrm{sec})$ & $5.0 \pm 0.6$ & $4.4 \pm 0.9$ & 0.013 \\
\hline Distal latency (sec) & $6.3 \pm 0.6$ & $7.1 \pm 0.8$ & 0.003 \\
\hline
\end{tabular}

${ }^{a}$ Paired $t$ test, compared with the baseline values.

LES, lower esophageal sphincter.

Values are presented as mean \pm standard deviation.

\section{The Effect of Stress on Esophageal Motility}

During real stress, contractile front velocity of esophageal peristaltic contractions was significantly decreased (Fig. 2), and distal latency of esophageal peristaltic contractions was significantly increased (Fig. 3), compared with the baseline period. Those alterations were not observed during sham stress. The percentages of weak, failed, hyper-contractile, rapid, and premature contractions were not significantly altered during real stress and sham stress, compared with those measured in the baseline period. No significant changes in the mean LES resting pressure and distal contractile integral of esophageal contractions were observed during real stress and sham stress, compared with those measured in the baseline period (Table 2).

\section{The Effect of Stress on Gastroesophageal Reflux Parameters}

BET, EAET, BCT, and ACT measured during real stress did not differ compared with those parameters measured in the basal period. No significant alterations in BET, EAET, BCT, and ACT were observed during sham stress compared with those parameters measured in the basal period (Table 3).
Table 3. Parameters of Esophageal Impedance-pH Monitoring During Real and Sham Stress

\begin{tabular}{lccc}
\hline \multicolumn{1}{c}{ Parameters } & Baseline & Stress period & $P$-value \\
\hline Sham stress & & & \\
Total \% BET & $7.0 \pm 16.4$ & $8.0 \pm 14.0$ & 0.823 \\
Total \% distal EAET & 0 & 0 & 0.343 \\
Median BCT $(\mathrm{sec})$ & 0 & $1.0 \pm 3.2$ & 0.393 \\
Mean ACT $(\mathrm{sec})$ & 0 & 0 & 0.840 \\
Real stress & & & \\
Total \% BET & $3.0 \pm 4.8$ & $20.8 \pm 2.6$ & 0.289 \\
Total \% distal EAET & 0 & $985.7 \pm 519.1$ & 0.075 \\
Median BCT (sec) & 0 & $5.0 \pm 0.9$ & 0.120 \\
Mean ACT (sec) & $1.0 \pm 3.2$ & $6.6 \pm 1.1$ & 0.343 \\
\hline
\end{tabular}

${ }^{a}$ Paired $t$ test, compared with the baseline values.

BET, bolus exposure time; EAET, esophageal acid exposure time; BCT, bolus clearance time; $\mathrm{ACT}$, acid clearance time.

Values are presented as mean \pm standard deviation.

\section{Discussion}

In the present study, we have shown that acute psychological or mental stress affects esophageal motility in healthy volunteers. However, significant motor abnormalities of the esophagus failed to 
be induced. Moreover, gastroesophageal reflux parameters were not significantly altered by this type of stress. These findings suggest that acute mild stress is not able to play a major role in the generation of abnormal esophageal motility or the induction of gastroesophageal reflux in healthy humans. Therefore, the exacerbation of heartburn by stress is more likely to be attributed to other factors such as the increase of esophageal sensitivity to reflux.

Psychological comorbidity is common in patients with GERD and associated with the poor response to proton pump inhibitor treatment. ${ }^{19}$ The majority of patients who complain of heartburn believe that stress exacerbates their heartburn symptom. However, little research has been done to evaluate the effect of stress in exacerbating the symptoms of GERD. Data regarding the effect of stress on gastroesophageal reflux have shown controversial results. A previous study showed that cold stress decreases the postprandial rate of transient LES relaxations and reflux episodes in healthy humans. ${ }^{13}$ The findings of the present study failed to demonstrate this inhibitory effect of stress on gastroesophageal reflux. Although we expected the increase of reflux episodes and gastroesophageal reflux by acute stress, gastroesophageal reflux parameters were not significantly changed by acute stress. This finding suggests that the effect of stress in exacerbating the symptoms of GERD is not associated with motor abnormalities or the increase of gastroesophageal reflux. Evidence suggests that stressed individuals with GERD have increased symptom intensity, and heightened sensitivity to esophageal acid perfusion. ${ }^{10}$ These findings were not observed in healthy controls. Patients with GERD may be more vulnerable to stress events than healthy individuals. The reasons why abnormal motility and gastroesophageal reflux were not induced by acute stress in the present study may be because the study subjects included were healthy volunteers. Further investigation in patients with GERD is warranted. Actually, it has been reported that psychological stress enhances the perception of symptoms in the absence of increased reflux in patients with GERD.?

A number of stress models have been used in human studies. In choosing a stress model, it should be considered whether the stress is acute or chronic, and if it is physical or psychological. Serum cortisol levels vary depending on the stressor type. ${ }^{20}$ Thus, the gastrointestinal response to stress may be different according to the stressor type. Actually, it is reported that colonic motor responses may vary depending on psychological or physical stress. ${ }^{21}$ The dichotomous listening has been used as a mild psychological stressor. Although it is a mild stressor, reproducible results of the dichotomous listening on gut sensitivity and motility have been reported. ${ }^{10-12,14,17,21-23}$ In the present study, acute auditory and visual stress significantly altered the contractile front velocity and distal latency of esophageal peristaltic contractions, indicating that this type of stressor affects esophageal motility. However, it did not lead to the induction of abnormal motor patterns such as weak, failed, hyper-contractile, rapid, or premature contractions. Moreover, LES resting pressure and distal contractile integral of esophageal contractions were not significantly changed by this type of stressor. A previous study from our group regarding the effect of acute auditory stress on gastric motility failed to show the activation of hypothalamic-pituitary-adrenal axis by this stressor. ${ }^{17}$ The mild feature of the stressor may explain the reason why abnormal motor patterns were not induced by real stress in the present study. One of the advantages of the dichotomous listening is that it is relatively mild and produces little threat to the subject's safety. In the present study, the visual stress was added to the auditory stress. Watching horror movies makes subjects feel more stressed and anxious, ${ }^{24}$ and is known to be effective in inducing the psychological stress enough to decrease vagal activity. ${ }^{25}$ Therefore, dichotomous listening with horror pictures appears to increase stress, whereas relaxing music with peaceful and beautiful pictures are likely to reduce stress. The influence of acute stress on vagal activity may be associated with the change in contractile front velocity and distal latency of esophageal peristaltic contractions.

To our best knowledge, this is the first study to use HRM and esophageal impedance-pH monitoring in the evaluation of the effect of stress on esophageal motility and gastroesophageal reflux. In previous studies regarding this topic, conventional manometry and esophageal $\mathrm{pH}$ monitoring were used. HRM is superior to conventional manometry in many ways, and can assess the effect of acute stress on esophageal motility more accurately than conventional manometry. Furthermore, esophageal impedance-pH monitoring has advantages over esophageal $\mathrm{pH}$ monitoring in evaluating gastroesophageal reflux parameters. The weakness in techniques may result in incorrect results, which may explain the controversial results of previous studies. A previous study showed that shortterm psychological stress decreased esophagogastric junction pressures through inhibition of the LES in healthy subjects, ${ }^{26}$ whereas another study revealed that cold stress decreased the postprandial rate of transient LES relaxations and reflux episodes in healthy humans. We failed to confirm a significant change in esophagogastric junction pressure due to acute stress. This may be partly attributed to the discrepancy in the technique of motility measurement. Dichotomous listening is reported to increase the perception of stimuli in different regions of the human colon. ${ }^{27}$ On the other hand, the significant relationship between the presence of a severe, sustained 
life stress and increased severity of heartburn is also demonstrated. ${ }^{28}$ Therefore, further investigations using strong or different stressors are required to clarify the effect of stress on esophageal motility and gastroesophageal reflux. Moreover, studies in patients with GERD for the evaluation of the effect of stress in exacerbating the symptoms associated with gastroesophageal reflux are warranted.

One of the limitations of this study was that we did not evaluate the change of the functions of the autonomic nervous system and the hypothalamic-pituitary-adrenal axis. ${ }^{29}$ We did not measure serum cortical levels to avoid stress by blood sampling and interruption of the protocol by blood sampling or testing of the autonomic nervous system function. Another limitation was that we used acute psychological stressors alone. Further studies using various types of acute and chronic stressors are required. Third, the sex difference of enrolled participants may have affected the results. In the present study, more male participants were enrolled in the study than female participants. Males are less likely to be affected by stress than females.

In conclusion, acute auditory and visual stress seems to affect esophageal body motility. However, it does not induce significant motor abnormalities or increase the gastroesophageal reflux in healthy humans. Further investigation regarding the effect of stress in patients with GERD is warranted.

\section{Financial support: None.}

\section{Conflicts of interest: None.}

Author contributions: Kwang Jae Lee designed the protocol, conducted the study, collected and interpreted data, and drafted the manuscript; Hong Sub Lee conducted the study, collected and interpreted data, and drafted the manuscript; and Chung Kyun Noh conducted the study and collected data.

\section{References}

1. Mönnikes H, Tebbe JJ, Hildebrandt M, et al. Role of stress in functional gastrointestinal disorders. Evidence for stress-induced alterations in gastrointestinal motility and sensitivity. Dig Dis 2001;19:201-211.

2. Bhatia V, Tandon RK. Stress and the gastrointestinal tract. J Gastroenterol Hepatol 2005;20:332-339.

3. Stam R, Croiset G, Akkermans LM, Wiegant VM. Sensitization of the colonic response to novel stress after previous stressful experience. Am J Physiol 1996;271(5 Pt 2):R1270-R1273.

4. Tsukada F, Nagura Y, Abe S, Sato N, Ohkubo Y. Effect of restraint and footshock stress and norepinephrine treatment on gastric emptying in rats. Biol Pharm Bull 2003;26:368-370.

5. Jung YS, Kim MY, Lee HS, Park SL, Lee KJ. Effect of DA-9701, a novel prokinetic agent, on stress-induced delayed gastric emptying and hormonal changes in rats. Neurogastroenterol Motil 2013;25:254-259, e166.

6. Song EM, Jung HK, Jung JM. The association between reflux esophagitis and psychosocial stress. Dig Dis Sci 2013;58:471-477.

7. Johnston BT, McFarland RJ, Collins JS, Love AH. Effects of acute stress on oesophageal motility in patients with gastro-oesophageal reflux disease. Gut 1996;38:492-497.

8. Richter JE, Bradley LC. Psychophysiological interactions in esophageal diseases. Semin Gastrointest Dis 1996;7:169-184.

9. Wright CE, Ebrecht M, Mitchell R, Anggiansah A, Weinman J. The effect of psychological stress on symptom severity and perception in patients with gastro-oesophageal reflux. J Psychosom Res 2005;59:415424.

10. Fass R, Naliboff BD, Fass SS, et al. The effect of auditory stress on perception of intraesophageal acid in patients with gastroesophageal reflux disease. Gastroenterology 2008;134:696-705.

11. Anderson KO, Dalton CB, Bradley LA, Richter JE. Stress induces alteration of esophageal pressures in healthy volunteers and non-cardiac chest pain patients. Dig Dis Sci 1989;34:83-91.

12. Johnston BT, McFarland RJ, Collins JS, Love AH. Effect of acute stress on oesophageal motility in patients with gastro-oesophageal reflux disease. Gut 1996;38:492-497.

13. Penagini R, Bartesaghi B, Bianchi PA. Effect of cold stress on postprandial lower esophageal sphincter competence and gastroesophageal reflux in healthy subjects. Dig Dis Sci 1992;37:1200-1205.

14. Gonlachanvit S, Rhee J, Sun WM, Chey WD. Effect of acute acoustic stress on anorectal function and sensation in healthy humans. Neurogastroenterol Motil 2005;17:222-228.

15. McRae S, Younger K, Thompson DG, Wingate DL. Sustained mental stress alters human jejunal motor activity. Gut 1982;23:404-409.

16. Cherry C. Some experiments on the recognition of speech with one and two ears. J Acoust Soc Am 1953;25:975-979.

17. Lee HS, An YS, Kang J, Yoo JH, Lee KJ. Effect of acute auditory stress on gastric motor responses to a meal in healthy volunteers. J Gastroenterol Hepatol 2013;28:1699-1704.

18. Bredenoord AJ, Fox M, Kahrilas PJ, Pandolfino JE, Schwizer W, Smout AJ. Chicago classification criteria of esophageal motility disorders defined in high resolution esophageal pressure topography. Neurogastroenterol Motil 2012;24(suppl 1):57-65.

19. Mizyed I, Fass SS, Fass R. Review article: gastro-oesophageal reflux disease and psychological comorbidity. Aliment Pharmacol Ther 2009;29:351-358.

20. Dickerson SS, Kemeny ME. Acute stressors and cortisol responses: a theoretical integration and synthesis of laboratory research. Psychol Bull 2004;130:355-391.

21. Rao SS, Hatfield RA, Suls JM, Chamberlain MJ. Psychological and physical stress induce differential effects on human colonic motility. Am J Gastroenterol 1998;93:985-990. 
22. Denson TF, Spanovic M, Miller N. Cognitive appraisals and emotions predict cortisol and immune responses: a meta-analysis of acute laboratory social stressors and emotion inductions. Psychol Bull 2009;135:823853.

23. Dickhaus B, Mayer EA, Firooz N, et al. Irritable bowel syndrome patients show enhanced modulation of visceral perception by auditory stress. Am J Gastroenterol 2003;98:135-143.

24. Mattar L, Zeeni N, Bassil M. Effect of movie violence on mood, stress, appetite perception and food preferences in a random population. Eur J Clin Nutr 2015;69:972-973.

25. Yin J, Levanon D, Chen JD. Inhibitory effects of stress on postprandial gastric myoelectrical activity and vagal tone in healthy subjects. Neuro- gastroenterol Motil 2004;16:737-744.

26. Mittal RK, Stewart WR, Ramahi M, Chen J, Tisdelle D. The effects of psychological stress on the esophagogastric junction pressure and swallow-induced relaxation. Gastroenterology 1994;106:1477-1484.

27. Ford MJ, Camilleri M, Zinsmeister AR, Hanson RB. Psychosensory modulation of colonic sensation in the human transverse and sigmoid colon. Gastroenterology 1995;109:1772-1780.

28. Naliboff BD, Mayer M, Fass R, et al. The effect of life stress on symptoms of heartburn. Psychosom Med 2004;66:426-434.

29. Taché $Y$, Million M. Role of corticotropin-releasing factor signaling in stress-related alterations of colonic motility and hyperalgesia. J Neurogastroenterol Motil 2015;21:8-24. 